\title{
Iterative solution of a class of nonlinear Fredholm integral equations
}

Juarez S. Azevedo, Adson M. Rocha ${ }^{1}$

CETEC-UFRB, Centro, 44380-000, Cruz das Almas-BA, Brazil

Saulo P. Oliveira ${ }^{3}$

DMAT-UFPR and INCT-GP, 81531-980, Curitiba-PR, Brazil.

\begin{abstract}
We consider the numerical solution of functional integral equations, a particular class of nonlinear Fredholm integral equations, by the collocation method with piecewise linear basis functions. The nonlinear algebraic system arising from the spatial discretization is iteratively solved with a fixed-point algorithm.
\end{abstract}

Keywords. Collocation method; Fixed-point iteration; Fredholm integral equation.

\section{Introduction}

Two important classes of nonlinear integral equations, namely Hammerstein and Urysohn equations, have been widely studied from both analytical [4] and numerical [3] points of view. Urysohn integral equation is given as

$$
u(x)=g(x)+\int_{a}^{b} K(x, y, u(y)) d y, \quad x \in[a, b],
$$

whereas Hammerstein is a particular case of (1):

$$
u(x)=g(x)+\int_{a}^{b} K(x, y) f(x, u(y)) d y, \quad x \in[a, b] .
$$

This paper concerns a slightly different nonlinear integral equation,

$$
u(x)=g(x)+f(x, \mathcal{K}[u](x)), \quad x \in[a, b],
$$

known as a functional integral equation [5]. The operator $\mathcal{K}$, defined as

$$
\mathcal{K}[u](x)=\int_{a}^{b} K(x, y) u(y) d y,
$$

\footnotetext{
${ }^{1}$ juarez@ufrb.edu.br

2 adson@ufrb.edu.br

${ }^{3}$ saulopo@ufpr.br
} 
is the linear integral operator associated with the kernel $K$, with $K:[a, b] \times[a, b] \longrightarrow R$. Integral equations such as (3) have been recently employed to describe generalized PoissonBoltzmann distributions of ions in models for swelling porous media [8].

We consider the following conditions below

a. $g \in L^{1}([0,1])$.

b. $f:[0,1] \times \mathbb{R} \rightarrow \mathbb{R}$ satisfies Caratheodory hypotheses (i.e. $f$ is measurable with respect to $x \in[0,1]$, for all $y \in \mathbb{R}$, and continuous in $y \in \mathbb{R}$, for a.a. $x \in[0,1])$ and there are $\theta \in L^{1}([0,1]), \tau \geq 0$ such that $f(x, y) \leq \theta(x)+\tau|y|, \quad x \in[0,1], \quad y \in \mathbb{R}$.

c. $K$ verifies Caratheodory hypotheses and there is $\gamma \in L^{1}([0,1])$ such that $K(x, y) \leq$ $\gamma(x), \quad x$ a.e. in $[0,1], \quad y \in \mathbb{R}$.

d. $\tau\|\mathcal{K}\|_{L^{1}}<1$.

Theorem 1.1. Under the assumptions (a)-(d) above the eq. (3) has at least one solution $u \in L^{1}([0,1])$ (see $\left.[7]\right)$.

Although the theorem is stated for the interval $[0,1]$, we can extend it through a change of variables to the form (3).

Few authors have considered the numerical approximation of functional integral equations $[1,9]$, motivating the present work to use the strategy known as fixed-point (Picard) iteration [2], together with the collocation discretization, to find an approximate solution $u^{(k)}$ to a solution of eq. (3).

The remainder of the paper is organized as follows. In Section 2 we present spatial discretization on collocation method and fixed-point scheme. Numerical results are provided in Section 3. Finally we draw some conclusions.

\section{Domain discretization and fixed-point method}

For simplicity, let us consider a uniform grid

$$
a=x_{0}<x_{1}<\ldots<x_{n}=b
$$

with spacing $h$, and let $\phi_{j} \in P_{1}([a, b])$ be the Lagrange global piecewise linear functions satisfying $\phi_{j}\left(x_{i}\right)=\delta_{i j}$. The collocation method for (3) with continuous piecewise linear functions reads as: find

$$
u_{h}(x)=\sum_{j=0}^{n} u_{j} \phi_{j}(x)
$$

such that

$$
u_{h}\left(x_{i}\right)=g\left(x_{i}\right)+f\left(x, \mathcal{K}\left[u_{h}\right]\left(x_{i}\right)\right), \quad 0 \leq i \leq n .
$$

It follows from the linearity of the operator $\mathcal{K}$ that

$$
u_{i}=g\left(x_{i}\right)+f\left(x_{i}, \sum_{j=0}^{n} \mathcal{K}\left[\phi_{j}\right]\left(x_{i}\right) u_{j}\right), \quad 0 \leq i \leq n .
$$


We employ the trapezoidal rule at the grid points to evaluate $\mathcal{K}\left[\phi_{j}\right]\left(x_{i}\right)$ :

$$
\mathcal{K}\left[\phi_{j}\right]\left(x_{i}\right)=\int_{a}^{b} K\left(x_{i}, y\right) \phi_{j}(y) d y \approx \sum_{k=0}^{n} w_{k} K\left(x_{i}, x_{k}\right) \phi_{j}\left(x_{k}\right)=w_{j} K\left(x_{i}, x_{j}\right),
$$

where $w_{0}=w_{n}=h / 2$ and $w_{k}=h(1 \leq k \leq n-1)$. This leads to the following numerical scheme: find $\boldsymbol{u}=\left[u_{0}, \ldots, u_{n}\right]^{T}$ such that

$$
\boldsymbol{u}=\mathcal{T}_{1}(\boldsymbol{u}), \quad \mathcal{T}_{1}(\boldsymbol{u})=\boldsymbol{g}+\boldsymbol{F}(\boldsymbol{x}, \tilde{K} \boldsymbol{u}),
$$

where $\tilde{K}, \boldsymbol{g}$, and $\boldsymbol{F}(\boldsymbol{x}, \boldsymbol{y})$ are defined as

$$
\tilde{K}_{i, j}=w_{j} K\left(x_{i}, x_{j}\right), \quad g_{i}=g\left(x_{i}\right), \quad F_{i}(\boldsymbol{x}, \boldsymbol{y})=f\left(x_{i}, y_{i}\right) .
$$

Note that, in general, $\tilde{K}$ is a dense matrix.

One strategy to solve (6) is to define $\boldsymbol{v}=\tilde{K} \boldsymbol{u}$. If $\tilde{K}$ is invertible, then

$$
\begin{gathered}
\tilde{K}^{-1} \boldsymbol{v}=\boldsymbol{F}(\boldsymbol{x}, \boldsymbol{v})+\boldsymbol{g} \\
\boldsymbol{v}=\tilde{K} \boldsymbol{F}(\boldsymbol{x}, \boldsymbol{v})+\tilde{K} \boldsymbol{g} .
\end{gathered}
$$

Thus, we first solve the nonlinear system

$$
\boldsymbol{v}=\mathcal{T}_{2}(\boldsymbol{v}), \quad \mathcal{T}_{2}(\boldsymbol{v})=\tilde{K} \boldsymbol{F}(\boldsymbol{x}, \boldsymbol{v})+\tilde{K} \boldsymbol{g},
$$

and afterwards we solve the linear system

$$
\tilde{K} \boldsymbol{u}=\boldsymbol{v} .
$$

Both (6) and (8) naturally lead to a fixed-point (Picard) iteration scheme:

$$
\boldsymbol{u}^{(k+1)}=\mathcal{T}_{1}\left(\boldsymbol{u}^{(k)}\right), \text { or } \boldsymbol{v}^{(k+1)}=\mathcal{T}_{2}\left(\boldsymbol{v}^{(k)}\right) .
$$

\section{$3 \quad$ Numerical example}

As a preliminary assessment of the methodology presented in this work, we consider the nonlinear integral equation (3) with $K(x, y)=e^{\lambda|x-y|}$ and $f(x, y)=\alpha y^{2}$, where $\lambda<0$ and $\alpha>0$. Here $g(x)$ is chosen as

$$
g(x)=-\alpha \cos ^{2}(x)+\left(2 \alpha-\frac{1+\lambda^{2}}{2 \lambda}\right) \cos (x)+\left(\frac{\lambda}{2}-\alpha\right),
$$

so that the exact solution on interval $[0,4 \pi]$ is given by

$$
u(x)=\frac{1}{2 \lambda}\left[-\left(1+\lambda^{2}\right) \cos (x)+\lambda^{2}\right] .
$$

The parameter $\lambda$ is related to the correlation length of the kernel. If $|\lambda|$ is large, then $K(x, y)$ decays more rapidly to zero as $|x-y|$ increases, which demands a finer 
discretization. On the other hand, $\alpha$ controls the nonlinearity of the problem in the sense that it weights $f$ in $(3)$.

Let $k$ the number of iterations of the Picard's method and $n$ the amount of point in the grid of the collocation method. In the following, we study how relative error depends of $k, n$, and consider the input parameters $\lambda$ and $\alpha$. In all tests we employ the initial guess $u_{0}(x)=2$ on the point-fixed method.

Let us start with the dependence on $k$, which is depicted in Fig. 1. We consider $\lambda=-10, n=50,100,500,1000$, and $\alpha=0.1,1$. We see from Figs. 1(a) and 1(b) that Picard's method converges to an approximate solution whose error decreases with the grid size, but it may not converge if the grid is not sufficiently refined (for example, when $n=50$ in Fig. 1(b)).

In Fig. 2, we let vary the grid parameter $n$ for $k=2,10,50$, and 100 iterations. In all cases a large number of points influenced in error decay although a slight divergence is observed in case $\alpha=1$ (see Fig. 2(b)).

In Fig. 3, we study the behavior of $\lambda$ on interval $[-10,-1]$ considering a fixed mesh given by $n=50$ and $n=500$ points respectively, and again admitting $k=2,10,50$, and 100 iterations for each analysis. Fig. 3(a) ensures that we are properly providing convergent solutions for sufficiently large number of iterations. On the other hand, as shown in Fig. 3(b), divergence may occur even in a fine mesh if $k$ is too small.

Fig. 4 examines the impact of the choice $\alpha$ on values present in the interval [0,3], following the same model as the previous figure. The comparisons show that when parameter $\alpha>1$ there is a divergence in the solution even when the refinement is more emphasized.

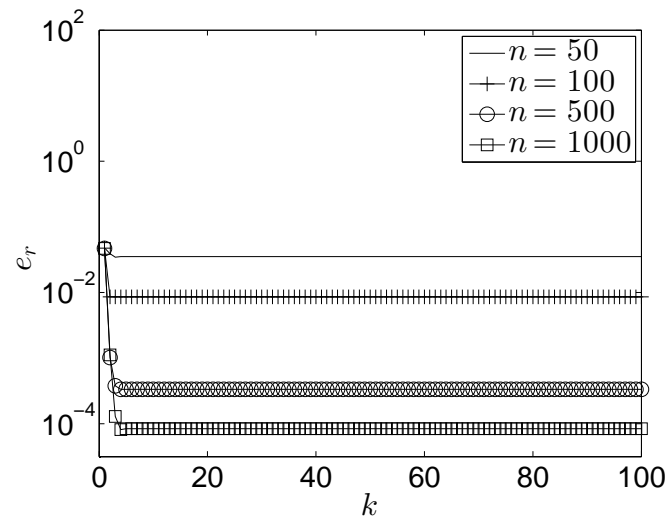

(a) $\lambda=-10$ and $\alpha=0.1$

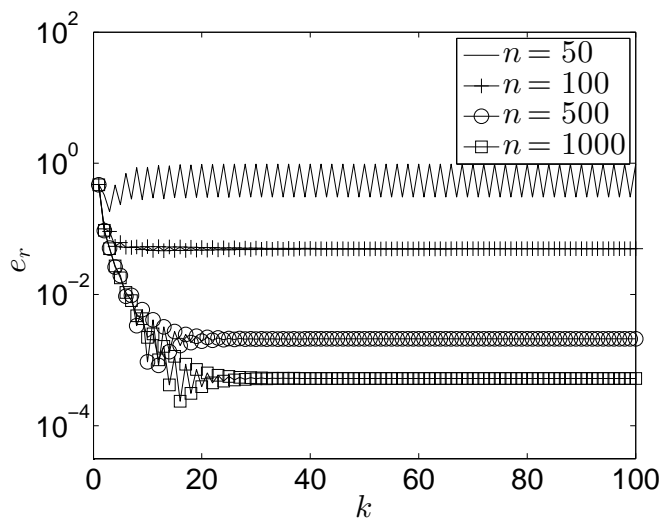

(b) $\lambda=-10$ and $\alpha=1$

Figure 1: Relative error in terms of the number of iterations $k$.

Finally, we estimate the order of accuracy $p$ associated with the grid spacing $h$. Initially, we consider the relationship $e_{k} \approx C h_{k}^{p}$, where $h_{k}=(b-a) / n_{k}$, and compute $p$ as follows:

$$
p_{k} \approx \frac{\log \left(e_{k} / e_{k-1}\right)}{\log \left(h_{k} / h_{k-1}\right)}
$$




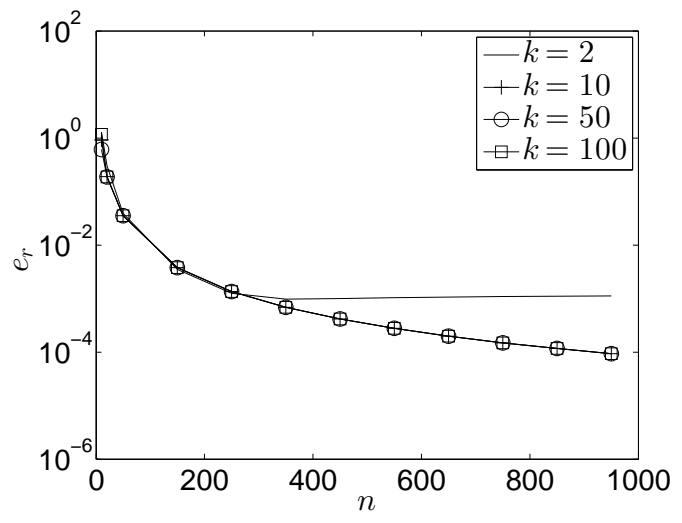

(a) $\lambda=-10$ and $\alpha=0.1$

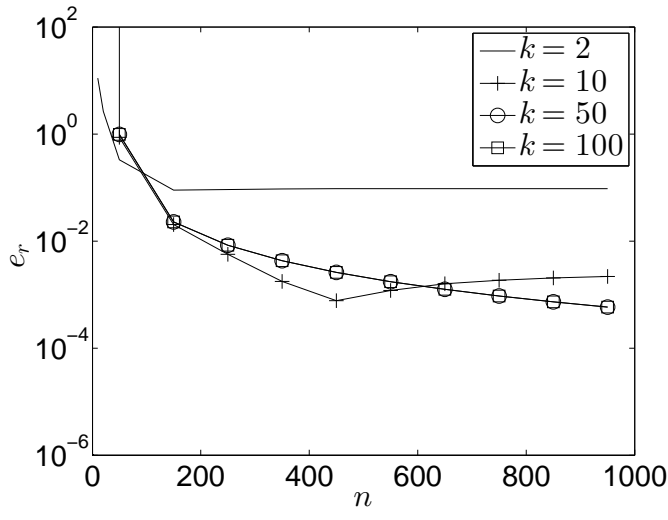

(b) $\lambda=-10$ and $\alpha=1$

Figure 2: Relative error in terms of the grid parameter $n$.

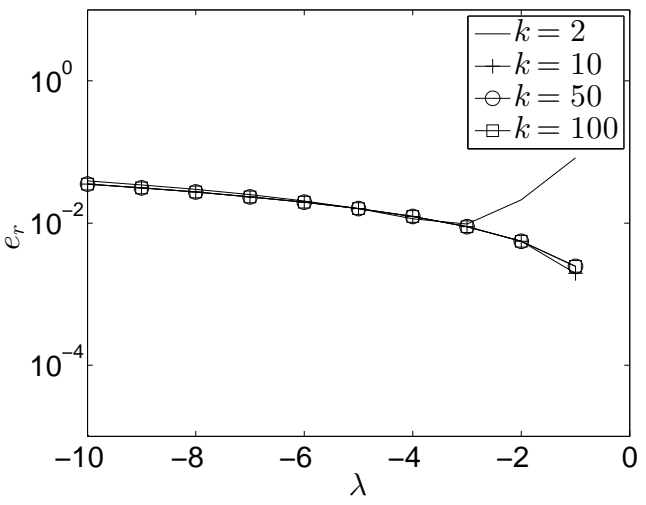

(a) $n=50$

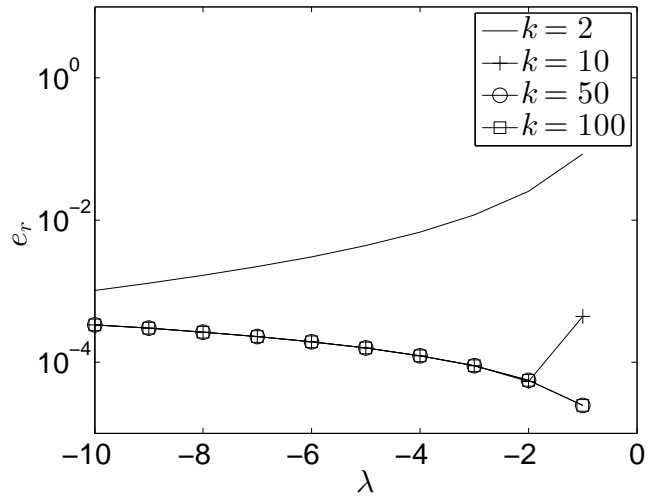

(b) $n=500$

Figure 3: Relative error in terms of the parameter $\lambda$. We employ $\alpha=0.1$.

where $e_{k}=\left\|u_{k}-u_{e x}\right\| /\left\|u_{e x}\right\|$ is the real relative error. However, following de Vahl Davis [6] and Roache [10], we can estimate the order of accuracy $p$ associated with the grid spacing $h_{k}$ without resorting to the exact solution:

$$
\tilde{p}_{i, k}=\frac{\log \left(\left|u_{k-2}\left(x_{i}\right)-u_{k-1}\left(x_{i}\right)\right| /\left|u_{k-1}\left(x_{i}\right)-u_{k}\left(x_{i}\right)\right|\right)}{\log (\zeta)} .
$$

Herein, $\zeta$ is the refinement ratio, which must remain constant (i.e., $h_{k-1} / h_{k}=\zeta \forall k$ ). Since this estimate depends on the grid point $x_{i}$, we consider the lowest one:

$$
\tilde{p}_{k}=\min _{0 \leq i \leq n_{k-2}} \tilde{p}_{i, k} .
$$

In Table 1 we compare the orders of accuracy obtained in (11) and (13). Both estimates suggest that the asymptotic order of accuracy is 2 . 


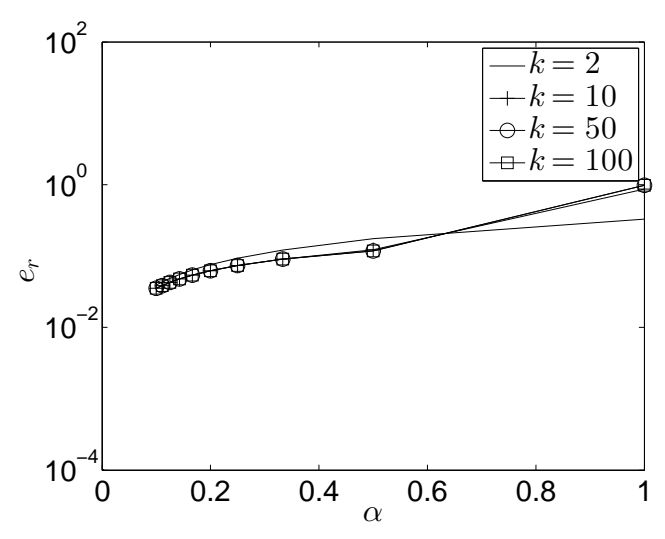

(a) $n=50$

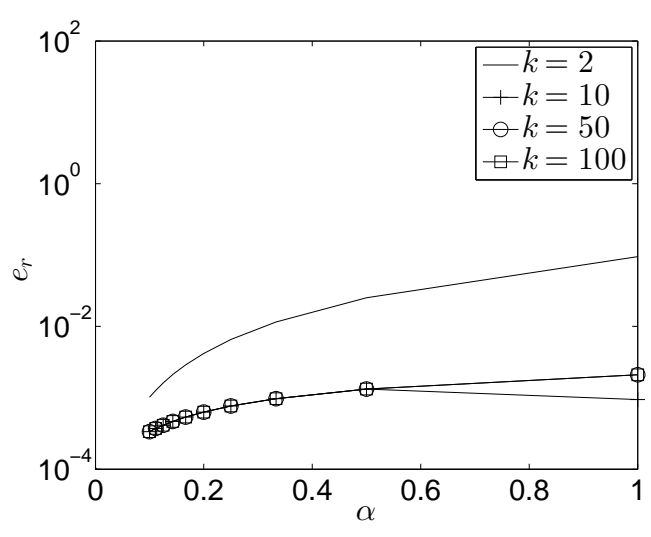

(b) $n=500$

Figure 4: Relative error in terms of the parameter $\alpha$. We employ $\lambda=-10$.

The results confirm that applying the simple trapezoidal rule to a subdivision of the original interval of integration, we have a convergence with order $\mathcal{O}\left(h^{2}\right)$.

\section{Conclusions}

An efficient and accurate numerical scheme based on the fixed-point and collocation methods was proposed for solving a nonlinear Fredholm integral equation of second kind. The numerical examples show that the accuracy improves with increase of $n$ and iterations number $k$. However, the variation in the parameters with $\lambda$ and $\alpha$ (at fixed $n$ ) gives an indication of how rapidly the solution curves are converging towards one another or diverging away from one another. In the example considered herein, the convergence rate of error is $\mathcal{O}\left(h^{2}\right)$, but this rate may drop depending on the smoothness of the input data. Theoretical verification of these error estimates is currently under study by the authors and will be the subject of future work.

\section{Acknowledgements}

This work was supported by Fundação Araucária (Project n. 39.591) and CNPq (grants 441489/2014-1 and 306083/2014-0).

\section{References}

[1] H. Adibi and A. Rismani. Numerical solution to a functional integral equations using the Legendre-spectral method. Aust. J. Basic Appl. Sci., 4(3):481-486, 2010.

[2] K. Atkinson. The numerical solution of Fredholm integral equations of the second kind with singular kernels. Numer. Math., 19, 1972. 
Table 1: Estimate the order $p$ based on (11) and (13) considering $\lambda=-10$ and $\alpha=0.1$ and with $k=10$ iterations. Below $e_{n}$ is real error, $p$ is the order obtained by (11) using the norm $\|\cdot\|_{\infty}$ and $\tilde{p}$ is the order obtained by (13).

\begin{tabular}{|c|c|c|c|c|}
\hline \hline$n$ & $h$ & $e_{n}$ & $p$ & $\tilde{p}$ \\
\hline 10 & 1.25664 & $9.17830 \mathrm{e}-01$ & 0.00000 & 0.00000 \\
\hline 20 & 0.62832 & $1.88984 \mathrm{e}-01$ & 0.00000 & 0.00000 \\
\hline 40 & 0.31416 & $5.50435 \mathrm{e}-02$ & 1.75364 & 1.43159 \\
\hline 80 & 0.15708 & $1.34880 \mathrm{e}-02$ & 2.01678 & 1.67561 \\
\hline 160 & 0.07854 & $3.30804 \mathrm{e}-03$ & 2.02465 & 2.02772 \\
\hline 320 & 0.03927 & $8.21935 \mathrm{e}-04$ & 2.00816 & 2.03350 \\
\hline 640 & 0.01963 & $2.05148 \mathrm{e}-04$ & 2.00218 & 2.01098 \\
\hline 1280 & 0.00982 & $5.12657 \mathrm{e}-05$ & 2.00055 & 2.00293 \\
\hline 2560 & 0.00491 & $1.28151 \mathrm{e}-05$ & 2.00014 & 2.00074 \\
\hline
\end{tabular}

[3] K. E. Atkinson. A survey of numerical methods for solving nonlinear integral equations. J. Integral Equations Appl., 4(1):15-46, 1992.

[4] J. Banaś. Integrable solutions of Hammerstein and Urysohn integral equations. $J$. Austral. Math. Soc. Ser. A, 46(1):61-68, 1989.

[5] J. Banaś and Z. Knap. Integrable solutions of a functional-integral equation. Rev. Mat. Univ. Complut. Madrid, 2(1):31-38, 1989.

[6] G. de Vahl Davis. Natural convection of air in a square cavity: a bench mark numerical solution. Int. J. Numer. Methods Fluids, 3(3):249-264, 1983.

[7] G. Emmanuele. About the existence of integrable solutions of a functional-integral equation. Rev. Mat. Univ. Complut. Madrid, 4(1):65-69, 1991.

[8] T. Le, C. Moyne, M. Murad, and S. Lima. A two-scale non-local model of swelling porous media incorporating ion size correlation effects. J. Mech. Phys. Solids, 61(12):2493-2521, 2013.

[9] K. Maleknejad, R. Mollapourasl, and P. Mirzaei. Numerical solution of Volterra functional integral equation by using cubic B-spline scaling functions. Numer. Methods Partial Differ. Equations, 30(2):699-722, 2014.

[10] P. Roache. Verification and validation in computational science and engineering. Hermosa, Albuquerque, 1998. 